\title{
INVESTIGACIONES
}

\section{Relaciones entre concepciones y prácticas pedagógicas: análisis de 13 Secuencias Didácticas de profesores de Historia usando tecnologías en el aula escolar*}

\author{
Relations between conceptions and pedagogical practices: \\ analysis of 13 Didactic Sequences of History teachers using technologies \\ in the school classroom
}

\author{
Dr. Marcelo Arancibia Herrera ${ }^{a}$, Dr. Alberto Galaz Ruíz ${ }^{b}$ \\ ${ }^{a}$ Profesor Jornada Completa Universidad Austral de Chile \\ Correo electrónico: marceloarancibia@uach.cl \\ ${ }^{\text {b }}$ Profesor Jornada Completa Universidad Austral de Chile \\ Correo electrónico: alberto.galaz@uach.cl
}

\section{RESUMEN}

Este artículo profundiza en el conocimiento sobre las relaciones entre las concepciones de profesores de Historia sobre enseñar y aprender con el uso didáctico de TIC. Fueron aplicadas entrevistas en profundidad y se analizaron secuencias didácticas con uso de Tecnología de 13 profesores de Historia. El tipo de análisis fue fenomenográfico configurándose categorías y subcategorías en relación a las dimensiones curriculares, didácticas y sobre el uso de TIC. Los resultados establecen patrones entre las concepciones del profesor con las decisiones didácticas en el aula cuando usa Tecnología. Se logra configurar en las secuencias didácticas Segmentos de Actuación Docente con uso de Tecnología asociados a concepciones de tipo Transmisiva, Constructivista Individual o Social. También, emerge la necesidad de instalar en la formación del profesorado el trabajo sobre los procesos de construcción de concepciones constructivistas como sustrato de la innovación didáctica con tecnología.

Palabras clave: Uso didáctico de tecnologías, concepciones del profesor de historia, Prácticas Pedagógicas, Aprendizaje y Enseñanza

\section{ABSTRACT}

This paper deepens into the understanding of relationships between History teachers' conceptions about teaching and learning through the didactic use of ICT. It is possible to find the submission of in-depth interviews and analysis of didactic sequences where 13 History teachers used technology. There was a phenomenographic analysis, which set categories and subcategories related to curricular, didactic and practical use dimensions when ICT were in use. Results evidence patterns between teacher's conceptions and pedagogical classroom decisions towards the use of technology in class. It is possible to establish segments of teacher's actions related to direct instruction or individual or social construction of knowledge within didactic sequences. Additionally, there is a need to include the processes of construction processes of constructivist conceptions basis for didactic innovation through ICT in the formation of teachers.

Key words: Didactic use of technologies, teacher's conceptions, Pedagogical Practices; Learning and teaching 
Estudios Pedagógicos XLV, N $^{\circ}$ 1: 103-121, 2019

RELACIONES ENTRE CONCEPCIONES Y PRÁCTICAS PEDAGÓGICAS: ANÁLISIS DE 13 SECUENCIAS DIDÁCTICAS DE PROFESORES DE HISTORIA USANDO TECNOLOGÍAS EN EL AULA ESCOLAR

\section{ENSEÑAR HISTORIA Y USO DE TECNOLOGÍAS}

La historia es una ciencia social cuyo objeto de estudio es el pasado de la humanidad (Bloch, 1975; Collingwood, 1977). Producto de esta definición, la historia de la historia presenta momentos de fuertes disputas respecto de cómo se ha de realizar el estudio del pasado (cuestiones de orden metodológico) y qué es el pasado y qué importancia tiene estudiarlo (cuestiones de orden epistemológicas) según Friera (1995) y Pagés (1983). Se trata de disputas disciplinares que contraerán tensiones expresadas, por ejemplo, en interrogantes tales como ¿Qué? y ¿Cómo debe enseñarse historia en contextos escolares? y que de una u otra forma nos reenvían a las diferencias existentes entre las dos corrientes de pensamiento que han primado en el estudio de la historia: el positivismo y el materialismo (Friera, 1995).

Por su parte, Camillioni (1994) afirma que la didáctica de la historia no solo tiene como objeto de estudio la práctica de la enseñanza de la disciplina, sino también los ámbitos de la reflexión sobre dicho quehacer. Existen tres ópticas respecto de cómo y para qué enseñar historia en educación secundaria (Estepa, 2000). En primer lugar, el enfoque instrumental o técnico orientado por una perspectiva que define la historia como una sucesión de acontecimientos secuenciales que deben ser conocidos por los estudiantes, tal como han sido relatados por la historiografía. Desde esta perspectiva, enseñar historia sería contar un relato sobre lo ocurrido que, con posterioridad, los estudiantes repiten en un relato con mayor o menor similitud. En segundo lugar, el enfoque ciudadano o práctico señala que enseñar historia es formar en los jóvenes conocimientos pragmáticos para que estos se configuren como buenos ciudadanos. De esta forma, más que poner énfasis en los relatos historiográficos del pasado, es primordial mirar cómo, a través de la historia, se ha conformado nuestro presente rescatándolo como elemento fundamental. En tercer lugar, el enfoque emancipatorio o crítico supone que la historia es un fenómeno de construcción social, muchas veces escrito de manera sesgada, por tanto, ideológicamente parcial. Enseñar historia es, en consecuencia, ayudar a descubrir que cada uno puede escribir su propia historia en relación con su colectividad, por tanto, el centro está puesto en la construcción de un sujeto consciente de su historicidad y condición social.

Además de cultivar y construir estructuras sobre el pasado y el presente la enseñanza de la historia se plantea como finalidad enseñar a pensar a sus estudiantes y mejorar su comprensión de cómo se construye el conocimiento histórico (Yilmaz, 2010). Este enfoque tiene como premisa que el conocimiento de la historia necesita ser contextualizado (Haydn, 2011). Una contextualización que a nuestro juicio alude al desarrollo de un proceso de mejoramiento didáctico en la enseñanza de la Historia y que, en el actual contexto educativo, parece ineludible asociarlo a la innovación con uso de TIC (Ertmer, OttenbreitLeftwich, 2010; Mueller, Wooda, Willoughby, Ross y Specht 2008; Starkey, 2011).

El contínuum de concepciones de los profesores sobre el significado de aprender también es bien conocido. En un extremo, el aprendizaje es copia de una realidad externa que ocurre en la mente de los estudiantes. En el otro extremo, el aprendizaje es un proceso de construcción que transcurre en la relación pedagógica entre profesor-estudiantecontenido. Esta dicotomía es ampliamente aceptada en la literatura sobre las concepciones de aprendizaje (Tigchelaar, Vermunt y Brouwer, 2012).

Por otro lado, la enseñanza de la historia, además de cultivar y construir estructuras sobre el pasado, plantea enseñar a pensar a sus estudiantes y mejorar su comprensión de 
cómo se construye el conocimiento histórico (Yilmaz, 2010). Este enfoque tiene como premisa que el conocimiento de la historia necesita ser contextualizado (Haydn, 2011).

Nuestra postura amplía la visión que reduce la innovación con TIC al aumento de cobertura y capacitación de profesores, diversificando la mirada hacia el uso de las TIC para desarrollar competencias digitales en los estudiantes, apropiadas a los comportamientos y sucesos conformes con la nueva centuria y el contexto globalizado (Gutiérrez y Tyner, 2012).

De esta forma, siguiendo a Ertmer y Ottenbreit-Leftwich (2010), para comprender y apoyar el uso eficiente de las TIC en las escuelas hay que atender al menos a cuatro fenómenos: el conocimiento docente sobre las tecnologías, la auto-eficacia de la TIC en sus prácticas, las concepciones pedagógicas que construyen los docentes sobre aprender y enseñar y, por último, la cultura escolar que favorece o dificulta esta incorporación. Con ello, la innovación educativa con TIC ha de atender a estos cuatro elementos sustantivos para que se instale, permanezca y provoque aprendizajes apropiados para la era digital.

Asimismo, la innovación didáctica con TIC no puede seguir reproduciendo prácticas que persiguen aprendizajes superficiales. Se debe avanzar hacia la obtención de aprendizajes profundos y de calidad, aprendizajes que exige una sociedad digital, orientados hacia habilidades de orden superior, tal cual las describe Starkey (2010 y 2011), quien construye una matriz en seis niveles de aprendizaje, los que asocia con prácticas educativas en que se usan TIC: 1) hacer, 2) estableciendo conexiones, 3) pensando sobre conceptos, 4) crítica y evaluación, 5) creación de conocimiento, y 6) compartiendo el conocimiento. Dado el carácter de las herramientas informáticas (principalmente la web social), estas facilitan y potencian que los estudiantes logren llegar al sexto nivel en el cual comparten el conocimiento que han construido a través de las plataformas sociales que ellos habitualmente usan.

Dado este análisis, los enfoques epistémicos sobre enseñar historia sustentados en las visiones del estudio histórico y la didáctica evidencian dilemas para el profesorado respecto de cómo enseñar, lo que repercute obviamente en las herramientas que deciden utilizar y cómo proponer su uso en clases a los estudiantes, pues esta decisión metodológica se sustenta en sus concepciones sobre aprender y enseñar la disciplina (Arancibia, Badia, Soto y Sigerson, 2018).

\section{CONCEPCIONES SOBRE APRENDER Y ENSEÑAR HISTORIA}

Hay consenso en la presencia de un contínuum de concepciones de los profesores sobre enseñar. En un extremo se sitúa la concepción tradicional que entiende la relación pedagógica como un traspaso de contenidos y conceptos centrados en el profesor. En el otro extremo una orientación innovadora centrada en el estudiante, focalizada en un protagonismo mayor de los alumnos (Petko, 2012; Tondeur, Hermans, Van Braak y Valcke, 2008). Por su parte, el contínuum de concepciones de los profesores sobre aprender también es bien conocido. Como ya se dijo, en un extremo se ubica el aprendizaje como copia de una realidad externa que ocurre en la mente de los estudiantes. En el otro extremo el aprendizaje es un proceso de construcción que transcurre en la relación pedagógica entre profesor-estudiante-contenido (Tigchelaar, Hermans, Van Braak y Valcke, 2012).

Las concepciones de los profesores sobre el conocimiento y el saber de la historia se centran mayoritariamente en un aprendizaje que permita responder pruebas escritas ya sea 
Estudios Pedagógicos XLV, N 1: 103-121, 2019

RELACIONES ENTRE CONCEPCIONES Y PRÁCTICAS PEDAGÓGICAS: ANÁLISIS DE 13 SECUENCIAS DIDÁCTICAS DE PROFESORES DE HISTORIA USANDO TECNOLOGÍAS EN EL AULA ESCOLAR

sobre memorización de datos o interpretación de fenómenos (Yilmaz, 2010). Evans (1994) ha descrito cinco tipos de profesores de historia que podemos resumir en tres grandes categorías: (1) narrador y filósofo cósmico, que se centra en historias sobre el pasado, patrones o grandes teorías; (2) historiador científico y relativista, que hace hincapié en la investigación para mejorar la comprensión de la historia o del presente; (3) maestro ecléctico, que presenta características de dos o más de las otras categorías.

Para Voet y de Wever (2016), los estudios sobre las creencias acerca de la naturaleza de la historia han adoptado a menudo una perspectiva de desarrollo y una visión del conocimiento como construcción contextual. Distinguen entre las creencias positivistas, haciendo hincapié en un informe neutral, distante y objetivo de los hechos históricos, y las creencias constructivistas, que argumentan que los hechos se interpretan inevitablemente por los historiadores, en la construcción de una narrativa personal del pasado. Como complemento a este dualismo Maggioni, VanSledright, y Alexander (2009) identifican creencias de los profesores como: (1) el objetivismo, que sostiene que la historia no tiene necesidad de la interpretación, sino que debe atenerse a las pruebas; (2) el subjetivismo, que insiste en que la historia es una interpretación, y que no hay evidencia real del pasado; o (3) criterialismo, que propone que la historia es una interpretación, pero debe estar basada en pruebas y argumentos. Del mismo modo, McCrum (2013) encontró concepciones en los profesores que denominó según la naturaleza de la disciplina como modernista o reconstruccionista, centrada en el profesor y con énfasis en la adquisición de contenidos; y postmodernas o construccionista, centrada en el estudiante y con énfasis en la interpretación e indagación.

En este continuo, Maggioni, VanSledright, y Alexander (2009) informan que la mayoría de los profesores están más cercanos a concepciones interpretativas, y en desacuerdo con las objetivistas. Por el contrario, McCrum (2013) encontró que los diferentes tipos de concepciones se extendieron casi uniformemente en un estudio con estudiantes de pedagogía. Por tanto, no se evidencia acuerdo sobre cómo se distribuyen las concepciones de los profesores de historia.

Desde una perspectiva general, se ha construido teóricamente una estructura categorial en tres ejes de concepciones sobre aprender y enseñar (Arancibia y Badia, 2015, p. 64).

A) Transmisiva/Reproductiva, donde el conocimiento es un objeto "traspasable", por tanto, posible de grabar en las mentes de otros individuos a través del acto de enseñar. Las concepciones están sustentadas en una realidad externa objetiva, su foco está puesto en un "objeto" que se ha de conocer. Por tanto, una concepción de tipo "Transmisiva".

B) Constructiva "individual", el aprendizaje es producto de una construcción interna, de naturaleza subjetiva. En tal caso, todo acto de enseñanza está supeditado a las motivaciones y estructuras previas o nivel de maduración cognitiva de los aprendices. Una enseñanza que busca un aprendizaje personal a través de procesos de asimilación y acomodación cercano al constructivismo cognitivo, que persigue nuevos equilibrios intrapsicológicos, con ello una construcción individual lo más cercana posible a la realidad externa.

C) Constructiva "social", entiende el proceso de naturaleza y origen del conocimiento como acto de aprendizaje recíproco, de construcción generado en interacción social, donde no existiría realidad externa sino conocimiento situado. El conocimiento es una trama o red de relaciones, donde se deconstruyen los roles 
instituidos. En este caso el foco está puesto en la relación, una concepción centrada en el proceso de aprender que tiene como base el constructivismo social, basado en la experiencia humana colectiva y en redes.

Poniendo en relación las perspectivas epistemológicas y las tradiciones didácticas expuestas con la estructura categorial de concepciones, es posible configurar tres tipos de práctica con uso de TIC asociadas a esta estructura categorial (Arancibia y Badia, 2013), sintetizadas en el Cuadro 1.

Cuadro 1. Prácticas sobre aprender y enseñar historia con TIC

\begin{tabular}{|c|c|c|c|}
\hline \multirow[t]{2}{*}{ Dimensión uso de TIC } & \multicolumn{3}{|c|}{ Tipo de Práctica de Enseñanza / Aprendizaje de la Historia } \\
\hline & $\begin{array}{l}\text { Transmisiva/ } \\
\text { Reproductiva }\end{array}$ & $\begin{array}{l}\text { Constructiva } \\
\text { "individual" }\end{array}$ & $\begin{array}{l}\text { Constructiva } \\
\text { "social" }\end{array}$ \\
\hline $\begin{array}{l}\text { Planificar el uso de la } \\
\text { TIC }\end{array}$ & $\begin{array}{l}\text { Complementar una } \\
\text { actividad }\end{array}$ & $\begin{array}{l}\text { Como actividad de los } \\
\text { estudiantes }\end{array}$ & $\begin{array}{l}\text { Profundizar en la } \\
\text { información }\end{array}$ \\
\hline $\begin{array}{l}\text { Tipo de uso educativo } \\
\text { del profesor }\end{array}$ & $\begin{array}{l}\text { Exponer contenido y } \\
\text { entregar información }\end{array}$ & $\begin{array}{l}\text { Mostrar ejemplo, } \\
\text { presentar contenidos }\end{array}$ & $\begin{array}{l}\text { Comunicativo, } \\
\text { simulaciones de } \\
\text { escenarios }\end{array}$ \\
\hline $\begin{array}{l}\text { Tipo de uso educativo } \\
\text { estudiantes }\end{array}$ & $\begin{array}{l}\text { Acceden y organizan } \\
\text { información de modo } \\
\text { restringido }\end{array}$ & $\begin{array}{l}\text { Buscan información, } \\
\text { realizan ejercicios }\end{array}$ & $\begin{array}{l}\text { Acceden a información, } \\
\text { comparten y discuten }\end{array}$ \\
\hline $\begin{array}{l}\text { Tipo de ayuda que } \\
\text { ofrece a sus estudiantes } \\
\text { y su papel durante el } \\
\text { trabajo }\end{array}$ & $\begin{array}{l}\text { Dar instrucciones, } \\
\text { supervisar buen manejo } \\
\text { del contenido, mantiene } \\
\text { distancia en el uso de } \\
\text { TIC }\end{array}$ & $\begin{array}{l}\text { Poca ayuda, deja que } \\
\text { los alumnos trabajen }\end{array}$ & $\begin{array}{l}\text { Mucha ayuda, respecto } \\
\text { de los procedimientos } \\
\text { de la tarea }\end{array}$ \\
\hline $\begin{array}{l}\text { Frecuencia de uso de los } \\
\text { computadores de parte } \\
\text { del profesor }\end{array}$ & $\begin{array}{l}\text { Alta. Las usa para } \\
\text { exponer contenido } \\
\text { buena parte de la clase }\end{array}$ & $\begin{array}{l}\text { Deja que la usen los } \\
\text { estudiantes de modo } \\
\text { muy dirigido }\end{array}$ & $\begin{array}{l}\text { La usan sus estudiantes } \\
\text { de manera libre }\end{array}$ \\
\hline $\begin{array}{l}\text { El tipo de software que } \\
\text { utilizan }\end{array}$ & $\begin{array}{l}\text { Básicamente software } \\
\text { de productividad para } \\
\text { dar respuesta a alguna } \\
\text { guía de aprendizajes o } \\
\text { cuestionario }\end{array}$ & Internet dirigido & Internet abierto \\
\hline $\begin{array}{l}\text { Las interacciones ante la } \\
\text { computadora }\end{array}$ & $\begin{array}{l}\text { Profesor - ordenador - } \\
\text { estudiantes }\end{array}$ & $\begin{array}{l}\text { Ordenador - estudiantes } \\
\text { - profesor }\end{array}$ & $\begin{array}{l}\text { Estudiantes - profesor } \\
\text { - ordenador }\end{array}$ \\
\hline Formación en TIC & $\begin{array}{l}\text { Aprender a usar las TIC } \\
\text { (recursos) }\end{array}$ & $\begin{array}{l}\text { Didáctica sobre el uso } \\
\text { de TIC }\end{array}$ & Entornos complejos \\
\hline $\begin{array}{l}\text { Dificultades en el uso de } \\
\text { TIC }\end{array}$ & $\begin{array}{l}\text { Los estudiantes saben } \\
\text { más que el profesor }\end{array}$ & $\begin{array}{l}\text { Bajo nivel de acceso a } \\
\text { computadores. }\end{array}$ & Alienación \\
\hline $\begin{array}{l}\text { Beneficios en el uso de } \\
\text { TIC }\end{array}$ & Acceso a información & $\begin{array}{l}\text { Motivación e } \\
\text { implicación en la } \\
\text { actividad para los } \\
\text { estudiantes }\end{array}$ & $\begin{array}{l}\text { Abre nuevos espacios y } \\
\text { ofrece otras } \\
\text { herramientas }\end{array}$ \\
\hline
\end{tabular}


Estudios Pedagógicos XLV, $\mathrm{N}^{\circ}$ 1: 103-121, 2019

RELACIONES ENTRE CONCEPCIONES Y PRÁCTICAS PEDAGÓGICAS: ANÁLISIS DE 13 SECUENCIAS DIDÁCTICAS DE PROFESORES DE HISTORIA USANDO TECNOLOGÍAS EN EL AULA ESCOLAR

Lo analizado, nos lleva a plantearnos la pregunta respecto de qué tipo de relación existe entre las concepciones de los profesores de Historia sobre aprender y enseñar con los tipos de usos educativos que despliega en sus clases con uso de TIC, dado que lo que nos refleja la evidencia es que según un tipo de concepción habría asociada un tipo de práctica que incide en la construcción de aprendizaje escolar (Arancibia, Badia, Soto y Sigerson, 2018; Inan, Lowther, Ross y Strahl, 2010).

\section{METODOLOGÍA}

Para intentar responder al problema planteado se propone un estudio de casos de tipo múltiple (Stake, 1999) organizado en dos etapas. Se aplican entrevistas semiestructuradas en profundidad y el registro en video de 13 Secuencias Didácticas (en adelante SD) de profesores de Historia en Chile, en cuatro ciudades (Valparaíso, Quilpué, Valdivia y Futrono) en dos regiones (Los Ríos y Valparaíso) y que se desempeñan en establecimientos educacionales de los tres tipos de dependencia administrativa que existe en Chile (Particular, Subvencionado y Municipal). A continuación, en el cuadro 2 se caracterizan los 13 casos:

Cuadro 2. Síntesis características socio profesionales "casos" (profesores)

\begin{tabular}{|c|c|c|c|c|c|}
\hline Profesor $^{1}$ & Edad & $\begin{array}{c}\text { Años } \\
\text { ejercicio }\end{array}$ & Formación profesional & Contexto desempeño & $\begin{array}{l}\text { Nivel uso } \\
\text { TIC }^{2}\end{array}$ \\
\hline P1 & 35 & 8 & $\begin{array}{l}\text { Profesora de Historia y } \\
\text { Geografía }\end{array}$ & $\begin{array}{l}\text { Subvencionado Laico } \\
\text { Valdivia }\end{array}$ & Avanzado \\
\hline $\mathbf{P 2}$ & 41 & 18 & $\begin{array}{l}\text { Profesora de Historia, } \\
\text { Geografía y Educación } \\
\text { cívica }\end{array}$ & $\begin{array}{l}\text { Particular } \\
\text { Valdivia }\end{array}$ & Intermedio \\
\hline P3 & 37 & 10 & $\begin{array}{l}\text { Profesora de Historia, } \\
\text { Geografía y Educación } \\
\text { cívica. }\end{array}$ & $\begin{array}{l}\text { Subvencionado } \\
\text { Religioso } \\
\text { Valdivia }\end{array}$ & Avanzado \\
\hline P4 & 32 & 5 & $\begin{array}{l}\text { Profesora de Historia y } \\
\text { Geografía }\end{array}$ & $\begin{array}{l}\text { Subvencionado Laico } \\
\text { Valdivia }\end{array}$ & Avanzado \\
\hline P5 & 33 & 5 & $\begin{array}{l}\text { Profesor de Historia y } \\
\text { Geografía }\end{array}$ & $\begin{array}{l}\text { Subvencionado } \\
\text { Religioso } \\
\text { Futrono } \\
\end{array}$ & Intermedio \\
\hline P6 & 38 & 10 & $\begin{array}{l}\text { Profesora de Historia, } \\
\text { Geografía y Educación } \\
\text { cívica }\end{array}$ & $\begin{array}{l}\text { Subvencionado } \\
\text { Religioso } \\
\text { Valdivia }\end{array}$ & Avanzado \\
\hline P7 & 37 & 7 & $\begin{array}{l}\text { Profesor de Historia, } \\
\text { Geografía y Educación } \\
\text { cívica }\end{array}$ & $\begin{array}{l}\text { Subvencionado Laico } \\
\text { Futrono }\end{array}$ & Intermedio \\
\hline P8 & 43 & 16 & $\begin{array}{l}\text { Profesora de Historia y } \\
\text { Geografía }\end{array}$ & $\begin{array}{l}\text { Subvencionado } \\
\text { Religioso } \\
\text { Futrono }\end{array}$ & Intermedio \\
\hline P9 & 42 & 13 & $\begin{array}{l}\text { Profesor de Historia, } \\
\text { Geografía y Educación } \\
\text { cívica }\end{array}$ & $\begin{array}{l}\text { Subvencionado Laico } \\
\text { Valdivia }\end{array}$ & Avanzado \\
\hline
\end{tabular}




\begin{tabular}{|c|c|c|l|l|l|}
\hline P10 & 42 & 19 & $\begin{array}{l}\text { Profesor de Historia, } \\
\text { Geografía y Educación } \\
\text { cívica }\end{array}$ & $\begin{array}{l}\text { Subvencionado } \\
\text { Municipal } \\
\text { Valdivia }\end{array}$ & Intermedio \\
\hline $\mathbf{P 1 1}$ & 28 & 5 & $\begin{array}{l}\text { Profesora de Historia y } \\
\text { Ciencias Sociales }\end{array}$ & $\begin{array}{l}\text { Subvencionado } \\
\text { Religioso } \\
\text { Valparaíso }\end{array}$ & Avanzado \\
\hline $\mathbf{P 1 2}$ & 51 & 27 & $\begin{array}{l}\text { Profesor de Historia y } \\
\text { Geografía }\end{array}$ & $\begin{array}{l}\text { Particular } \\
\text { Quilpue }\end{array}$ & Avanzado \\
\hline $\mathbf{P 1 3}$ & 47 & 17 & $\begin{array}{l}\text { Profesor de Historia, } \\
\text { Geografía y Educación } \\
\text { cívica }\end{array}$ & $\begin{array}{l}\text { Subvencionado } \\
\text { Religioso } \\
\text { Valparaíso }\end{array}$ & Intermedio \\
\hline
\end{tabular}

1 En adelante los profesores del estudio se denominarán con la letra P (mayúsculas) enumerados desde el 1 al 13, respectivamente.

2 Auto diagnóstico que realizaba cada profesor a través de un ítem en la entrevista que consideraba los siguientes niveles: Básico (maneja software de productividad de manera instrumental como por ejemplo hace documentos en Word, presentaciones y/o lista de notas en Excel, navega esporádicamente en internet). Intermedio (a lo anterior se le agrega que prepara materiales con uso de software e internet de apoyo a su docencia). Avanzado (Lo anterior pero además se comunica e interactúa por la red y sistemas informáticos con autonomía). Experto (todo lo anterior y además es capaz de enseñar a otros/as el uso de herramientas de productividad y apoyar a colegas en usos didácticos de las TIC).

Fuente: Actualizado de Arancibia, Soto y Casanova (2017, p.106).

La primera etapa de aplicación de una entrevista semiestructurada que, según Flick (2004), es un método clave para reconstruir teorías subjetivas, para lo cual debe recoger de los entrevistados los supuestos explícitos e inmediatos que se expresan casi espontáneamente en las respuestas a las preguntas definidas. A continuación, en el Cuadro 3 presentamos ejemplos de preguntas:

Cuadro 3. Ejemplos preguntas entrevistas

\begin{tabular}{|l|l|}
\hline $\begin{array}{l}\text { Parte 1. } \\
\text { Datos personales }\end{array}$ & $\begin{array}{l}\text { Año de obtención título: } \\
\text { Años de ejercicio docente: } \\
\text { ¿Cómo se ha formado en el uso de tic? }\end{array}$ \\
\hline $\begin{array}{l}\text { Parte 2. } \\
\text { Concepciones de } \\
\text { enseñanza y aprendizaje } \\
\text { de su disciplina }\end{array}$ & $\begin{array}{l}\text { ¿Cuál es el sentido que asigna usted a la enseñanza de su disciplina } \\
\text { en los establecimientos educacionales? } \\
\text { ¿Cómo piensa que aprenden sus alumnos? } \\
\text { ¿Con qué rasgos identifica una buena práctica docente? } \\
\text { ¿Qué consejos le daría usted a un profesor novel para afrontar su } \\
\text { inserción profesional? } \\
\text { ¿Cómo utiliza los resultados de sus evaluaciones? }\end{array}$ \\
\hline $\begin{array}{l}\text { Parte 3. } \\
\text { Concepciones sobre } \\
\text { aprender y enseñar con } \\
\text { TIC }\end{array}$ & $\begin{array}{l}\text { ¿En qué funciones cree han sido útiles las tic en su establecimiento } \\
\text { educativo? }\end{array}$ \\
¿En qué labores las utiliza usted? \\
¿Qué relevancia le otorga usted al uso de las tic?
\end{tabular}


Para el análisis de la entrevista se utiliza el enfoque fenomenográfico (Marton y Booth, 1997). Un enfoque cuyo propósito central es identificar las formas cualitativas con que las personas experimentan los fenómenos y comprenden la existencia de una relación objeto -sujeto. De esta forma las concepciones y formas de comprensión de los fenómenos no son vistas como cualidades individuales ni tampoco trata de determinar la naturaleza objetiva de cada una de ellas. Las transcripciones son analizadas en base a una matriz que contempló dimensiones, categorías y subcategorías:

- Dimensiones. Corresponde al agrupamiento de los temas en amplios bloques conceptuales que se han intencionado para que hablen los profesores durante la entrevista, relacionados con los aspectos temáticos abordados en la investigación, a saber: Dimensión 1 Curriculum, Dimensión 2 Didáctica y Dimensión 3 Uso de $\mathrm{TIC}^{1}$.

- Categorías: Diferenciaciones específicas de las dimensiones en aspectos que permitieron definir subtemas sobre los cuales hablan todos los profesores en las entrevistas.

- Subcategorías: diferentes niveles de concepciones entre los profesores en relación con una categoría. Corresponde a la expresión específica del conocimiento temático del profesor que permitirá clasificarlos y distinguirlos entre sí.

En una segunda etapa, la investigación se sustenta en la filmación de SD. Una SD corresponde a la unidad de análisis de la práctica docente con uso de TIC, en este caso cada profesor desarrolló una SD propuesta por él, entre 3 a 5 sesiones. Cada SD se analiza dividiendo y clasificando las sesiones en función de la identificación y descripción de diversos Segmentos de Actuación Docente (SAD), con ellos se construye una tipología que define tipos de usos educativos de TIC, en función de lo que hacen los profesores (SADP) y de las actividades que propone a los estudiantes (SADE). Este análisis de los videos permite identificar diversos $\mathrm{SAD}$, los que se definen como estructuras homogéneas de acción del docente o los estudiantes respecto del uso (o no uso) que le dan a las TIC durante una SD (Coll, Rochera y Colomina, 2010).

\section{RESULTADOS}

El cuadro 4 presenta la clasificación de los profesores por concepción preferente luego del análisis fenomenográfico de las entrevistas (dimensiones, categorías y subcategorías). Como allí se puede apreciar cuatro profesores fueron clasificados como Constructivista/ social, cuatro profesores Constructivista/individual y cinco profesores Transmisivo/ reproductivo. Esta distribución, lograda con el principio de saturación de los datos, nos permite tener un cuadro muy bien distribuido, para luego encontrar las asociaciones entre las prácticas con uso de TIC con las concepciones que tienen los trece profesores de Historia agrupados por concepción preferente. 
Estudios Pedagógicos XLV, $N^{\circ}$ 1: 103-121, 2019 RELACIONES ENTRE CONCEPCIONES Y PRÁCTICAS PEDAGÓGICAS: ANÁLISIS DE 13 SECUENCIAS DIDÁCTICAS DE PROFESORES DE HISTORIA USANDO TECNOLOGÍAS EN EL AULA ESCOLAR

Cuadro 4. Síntesis concepción preferente de cada profesor por dimensión

\begin{tabular}{|c|c|c|c|c|}
\hline & D1 & D2 & D3 & $\begin{array}{l}\text { Concepción } \\
\text { Preferente }\end{array}$ \\
\hline P1 & $\begin{array}{l}\text { Historia centrada en } \\
\text { el contexto, } \\
\text { curriculum crítico }\end{array}$ & $\begin{array}{l}\text { Enseñanza crítica que } \\
\text { permita la } \\
\text { socialización y } \\
\text { construcción de los } \\
\text { aprendizajes }\end{array}$ & $\begin{array}{l}\text { Las TIC elementos } \\
\text { motivadores y } \\
\text { transformadores }\end{array}$ & $\begin{array}{l}\text { Constructivista/ } \\
\text { social }\end{array}$ \\
\hline P2 & $\begin{array}{l}\text { Historia como } \\
\text { conocimiento } \\
\text { objetivo del pasado, } \\
\text { curriculum técnico }\end{array}$ & $\begin{array}{l}\text { Enseñanza } \\
\text { instrumental con } \\
\text { énfasis en la } \\
\text { reproducción objetiva } \\
\text { del contenido }\end{array}$ & $\begin{array}{l}\text { Las TIC apoyan la } \\
\text { enseñanza }\end{array}$ & $\begin{array}{l}\text { Transmisiva/ } \\
\text { reproductiva }\end{array}$ \\
\hline P3 & $\begin{array}{l}\text { Historia centrada en } \\
\text { el contexto } \\
\text { currículum crítico }\end{array}$ & $\begin{array}{l}\text { Enseñanza crítica que } \\
\text { permita la } \\
\text { socialización y } \\
\text { construcción de los } \\
\text { aprendizajes }\end{array}$ & $\begin{array}{l}\text { Las TIC como } \\
\text { complementos que } \\
\text { facilitan el acceso a } \\
\text { información }\end{array}$ & $\begin{array}{l}\text { Constructivista/ } \\
\text { social }\end{array}$ \\
\hline P4 & $\begin{array}{l}\text { Historia como } \\
\text { conocimiento } \\
\text { personal curriculum } \\
\text { práctico }\end{array}$ & $\begin{array}{l}\text { Enseñanza } \\
\text { instrumental con } \\
\text { énfasis en la } \\
\text { internalización y el } \\
\text { control }\end{array}$ & $\begin{array}{l}\text { Las TIC para } \\
\text { Infoalfabetizar }\end{array}$ & $\begin{array}{l}\text { Transmisiva/ } \\
\text { reproductiva }\end{array}$ \\
\hline P5 & $\begin{array}{l}\text { Historia como } \\
\text { conocimiento } \\
\text { objetivo del pasado, } \\
\text { curriculum técnico }\end{array}$ & $\begin{array}{l}\text { Enseñanza } \\
\text { instrumental con } \\
\text { énfasis en la } \\
\text { reproducción objetiva } \\
\text { del contenido }\end{array}$ & $\begin{array}{l}\text { Las TIC apoyan la } \\
\text { enseñanza }\end{array}$ & $\begin{array}{l}\text { Transmisiva/ } \\
\text { reproductiva }\end{array}$ \\
\hline P6 & $\begin{array}{l}\text { Historia centrada en } \\
\text { la planificación, } \\
\text { curriculum } \\
\text { instrumental }\end{array}$ & $\begin{array}{l}\text { Enseñanza eficiente } \\
\text { que permita } \\
\text { internalización y } \\
\text { verificación de los } \\
\text { aprendizajes }\end{array}$ & $\begin{array}{l}\text { Las TIC elementos } \\
\text { que estimulan y } \\
\text { permiten acceder a } \\
\text { información }\end{array}$ & $\begin{array}{l}\text { Constructivista/ } \\
\text { individual }\end{array}$ \\
\hline P7 & $\begin{array}{l}\text { Historia centrada en } \\
\text { el contexto } \\
\text { currículum crítico }\end{array}$ & $\begin{array}{l}\text { Enseñanza crítica que } \\
\text { permita la } \\
\text { socialización y } \\
\text { construcción de los } \\
\text { aprendizajes }\end{array}$ & $\begin{array}{l}\text { Las TIC apoyan la } \\
\text { enseñanza y } \\
\text { trasforman }\end{array}$ & $\begin{array}{l}\text { Constructivista/ } \\
\text { social }\end{array}$ \\
\hline P8 & $\begin{array}{l}\text { Historia como } \\
\text { conocimiento } \\
\text { autónomo, curriculum } \\
\text { instrumental }\end{array}$ & $\begin{array}{l}\text { Enseñanza eficiente } \\
\text { que permita } \\
\text { internalización y } \\
\text { verificación de los } \\
\text { aprendizajes }\end{array}$ & $\begin{array}{l}\text { Las TIC } \\
\text { complementan la } \\
\text { enseñanza y } \\
\text { estimulan }\end{array}$ & $\begin{array}{l}\text { Constructivista/ } \\
\text { individual }\end{array}$ \\
\hline
\end{tabular}


RELACIONES ENTRE CONCEPCIONES Y PRÁCTICAS PEDAGÓGICAS: ANÁLISIS DE 13 SECUENCIAS DIDÁCTICAS DE PROFESORES DE HISTORIA USANDO TECNOLOGÍAS EN EL AULA ESCOLAR

\begin{tabular}{|l|l|l|l|l|}
\hline P9 & $\begin{array}{l}\text { Historia centrada en } \\
\text { la planificación, } \\
\text { curriculum } \\
\text { instrumental }\end{array}$ & $\begin{array}{l}\text { Enseñanza eficiente } \\
\text { que permita } \\
\text { internalización y } \\
\text { verificación de los } \\
\text { aprendizajes }\end{array}$ & $\begin{array}{l}\text { Las TIC elementos } \\
\text { que estimulan y } \\
\text { permiten acceder a } \\
\text { información }\end{array}$ & $\begin{array}{l}\text { Constructivista/ } \\
\text { individual }\end{array}$ \\
\hline P10 & $\begin{array}{l}\text { Historia como } \\
\text { conocimiento } \\
\text { objetivo del pasado, } \\
\text { curriculum técnico }\end{array}$ & $\begin{array}{l}\text { Enseñanza } \\
\text { instrumental con } \\
\text { énfasis en la } \\
\text { reproducción objetiva } \\
\text { del contenido }\end{array}$ & $\begin{array}{l}\text { Las TIC apoyan la } \\
\text { enseñanza }\end{array}$ & $\begin{array}{l}\text { Transmisiva/ } \\
\text { reproductiva }\end{array}$ \\
\hline P11 & $\begin{array}{l}\text { Historia centrada en } \\
\text { el contexto } \\
\text { currículum crítico }\end{array}$ & $\begin{array}{l}\text { Enseñanza crítica que } \\
\text { permita la } \\
\text { socialización y } \\
\text { construcción de los } \\
\text { aprendizajes }\end{array}$ & $\begin{array}{l}\text { Las TIC } \\
\text { complementan la } \\
\text { enseñanza y } \\
\text { estimulan }\end{array}$ & $\begin{array}{l}\text { Constructivista/ } \\
\text { social }\end{array}$ \\
\hline P12 & $\begin{array}{l}\text { Historia centrada en } \\
\text { la planificación, } \\
\text { curriculum } \\
\text { instrumental }\end{array}$ & $\begin{array}{l}\text { Enseñanza eficiente } \\
\text { que permita } \\
\text { internalización y } \\
\text { verificación de los } \\
\text { aprendizajes }\end{array}$ & $\begin{array}{l}\text { Las TIC elementos } \\
\text { que estimulan y } \\
\text { permiten acceder a } \\
\text { información }\end{array}$ & $\begin{array}{l}\text { Constructivista/ } \\
\text { individual }\end{array}$ \\
\hline P13 & $\begin{array}{l}\text { Historia como } \\
\text { conocimiento } \\
\text { objetivo del pasado, } \\
\text { instrumental }\end{array}$ & $\begin{array}{l}\text { Enseñanza } \\
\text { instrumental con } \\
\text { énfasis en la } \\
\text { internalización y } \\
\text { verificación de los } \\
\text { aprendizajes }\end{array}$ & $\begin{array}{l}\text { Las TIC permiten } \\
\text { acceder a información } \\
\text { y para Infoalfabetizar }\end{array}$ & $\begin{array}{l}\text { Transmisiva/ } \\
\text { reproductiva }\end{array}$ \\
\hline
\end{tabular}

Fuente: Propia

A continuación, en los cuadros 5 y 6 se describen los SAD encontrados en las 13 SD, las que están constituidas por un total de 3134 minutos de video distribuidos en 49 sesiones de clases. El Cuadro 5, muestra $4 \mathrm{SAD}$ en que el profesor usa exclusivamente las TIC, codificados como SADP. En el Cuadro 5 se describen 7 SAD en los cuales los estudiantes utilizan las TIC, y se les asigna el código SADE. Si bien se observa que son más diversas las actividades en que las TIC son usadas por los estudiantes, en contraste a los segmentos en que las TIC son usadas por los profesores, sin embargo, en ambos no existe mucha diversidad. 
Cuadro 5. Descripción de categorías SAD con uso de TIC por parte del profesor (SADP)

\begin{tabular}{|c|c|}
\hline $\begin{array}{l}\text { Código y } \\
\text { nombre SAD }\end{array}$ & Descripción \\
\hline $\begin{array}{l}\text { SADP1 } \\
\text { Apoya la } \\
\text { exposición oral }\end{array}$ & $\begin{array}{l}\text { Segmento en el cual el profesor expone un contenido con apoyo de algún } \\
\text { recurso TIC. La principal fuente de información es su discurso y el recurso } \\
\text { tecnológico es solo un apoyo (visual y/o textual) a dicha exposición de } \\
\text { contenidos. Principalmente, son usadas TIC de presentaciones, específicamente } \\
\text { PowerPoint. El objetivo se centra en entregar información que los estudiantes } \\
\text { reciben pasivamente, aun cuando puede darse una modalidad mixta de } \\
\text { preguntas y respuestas, pero el principal foco es revisar contenidos. }\end{array}$ \\
\hline $\begin{array}{l}\text { SADP2 } \\
\text { Apoya la } \\
\text { conversación con } \\
\text { los alumnos }\end{array}$ & $\begin{array}{l}\text { Fragmento de la SD en que el profesor se apoya en algún recurso TIC para } \\
\text { generar un diálogo con los estudiantes, es decir, las TIC son base para generar } \\
\text { conversaciones sobre el contenido. Principalmente, el profesor extrae algún } \\
\text { recurso o material TIC y a partir de él genera alguna discusión o debate con } \\
\text { sus estudiantes donde lo que interesa es la reacción u opinión de los alumnos } \\
\text { respecto del material expuesto, y no tanto la información o contenido que el } \\
\text { profesor quiere transmitir, como sí sucede en el segmento anterior. La TIC } \\
\text { usada, por tanto, cumple una finalidad de activar una conversación a partir de } \\
\text { su contenido, en nuestros casos se observa este segmento cuando el profesor, } \\
\text { a partir de una elaboración de los propios estudiantes, detiene el proceso de } \\
\text { todo el curso y fomenta una discusión en torno al recurso hallado por un } \\
\text { grupo. }\end{array}$ \\
\hline $\begin{array}{l}\text { SADP3 } \\
\text { Muestra } \\
\text { ejemplos de } \\
\text { productos }\end{array}$ & $\begin{array}{l}\text { Corresponde a los momentos en que las TIC son usadas por el profesor para } \\
\text { ejemplificar los productos que espera sean desarrollados por los estudiantes, } \\
\text { es decir, en que muestra en pantalla productos "ideales" respecto de lo } \\
\text { solicitado en las tareas a desarrollar durante la secuencia didáctica. Por tanto, } \\
\text { las TIC sirven para modelar productos según lo que ha sido definido por el } \\
\text { profesor como ideal. }\end{array}$ \\
\hline $\begin{array}{l}\text { SADP4 } \\
\text { Presenta } \\
\text { contenidos } \\
\text { curriculares }\end{array}$ & $\begin{array}{l}\text { Segmentos de la SD en que el contenido está puesto en las TIC. De esta forma, } \\
\text { el profesor las usa para que los estudiantes adquieran la información } \\
\text { previamente preparada. En este caso el recurso TIC contiene y transmite el } \\
\text { contenido o información. Por ende, estos SAD suponen una presentación, a } \\
\text { toda la clase, con TIC de contenido sin mayor participación del profesor, a } \\
\text { través de algún video o PowerPoint automatizado en el que transcurre una } \\
\text { secuencia de información que el profesor comenta al principio, durante y/o al } \\
\text { final de la secuencia. El propósito del uso de la TIC está puesto en transmitir } \\
\text { información o bien motivar a los estudiantes con algún diaporama que presenta } \\
\text { información temática de la unidad. }\end{array}$ \\
\hline
\end{tabular}


Cuadro 6. Descripción de categorías SAD con uso de TIC de los estudiantes (SADE)

\begin{tabular}{|c|c|}
\hline $\begin{array}{l}\text { Código y } \\
\text { nombre SAD }\end{array}$ & Descripción \\
\hline $\begin{array}{l}\text { SADE1 } \\
\text { Acceder a } \\
\text { información } \\
\text { seleccionada }\end{array}$ & $\begin{array}{l}\text { SAD en que los estudiantes, a petición del profesor, se dirigen a buscar un } \\
\text { recurso o información específico que el profesor les indica de manera directa } \\
\text { para su revisión. El objetivo de las TIC está puesto en que los estudiantes } \\
\text { ingresan a algún recurso para extraer información, con la salvedad de que } \\
\text { este recurso es entregado directamente por el profesor, sin mayor proceso de } \\
\text { búsqueda por parte de los alumnos. }\end{array}$ \\
\hline $\begin{array}{l}\text { SADE2 } \\
\text { Visualizar } \\
\text { información }\end{array}$ & $\begin{array}{l}\text { En estos SAD las TIC son usadas por los estudiantes como un recurso para } \\
\text { observar algún tipo de material que el profesor solicita visualizar de manera } \\
\text { directa y explícita. El recurso visual expuesto se constituye en el foco del } \\
\text { segmento, el profesor tiene mínima o nula participación y los estudiantes } \\
\text { deben observar lo que va pasando por la pantalla, ya sea un diaporama en } \\
\text { PowerPoint o un video, por tanto, el recurso TIC expuesto contiene el } \\
\text { contenido en sí mismo. }\end{array}$ \\
\hline $\begin{array}{l}\text { SADE3 } \\
\text { Buscar } \\
\text { información }\end{array}$ & $\begin{array}{l}\text { Actividades en que, de modo preferente, los estudiantes buscan información } \\
\text { o recursos a través de Internet sin mayores restricciones de parte del docente. } \\
\text { Corresponde a momentos de las actividades en que los estudiantes navegan } \\
\text { de manera abierta, buscando información que almacenan y organizan sin } \\
\text { mayores indicaciones, a diferencia del segmento "elaboran un producto", } \\
\text { aquí el material o producto que han de crear o realizar a partir de la } \\
\text { información recopilada no es lo primordial, lo que interesa es la recolección } \\
\text { de información y queda muchas veces a la decisión de los estudiantes el } \\
\text { software o contenedor que usan para almacenar la información que van } \\
\text { obteniendo. Por tanto, el recurso principal aquí es la Web y el proceso se } \\
\text { centra en la navegación para encontrarinformación sobre los temas requeridos } \\
\text { por el profesor, cuya labor es supervisar o controlar que los estudiantes } \\
\text { lleguen a información confiable y adecuada. }\end{array}$ \\
\hline $\begin{array}{l}\text { SADE4 } \\
\text { Seguir } \\
\text { instrucciones }\end{array}$ & $\begin{array}{l}\text { Segmentos en que las TIC son usadas para hacer algo muy concreto con un } \\
\text { recurso TIC. El profesor indica los pasos y supervisa que los estudiantes } \\
\text { sigan la secuencia hasta llegar al lugar indicado o bien, realicen lo que les } \\
\text { pide. Por tanto, aquí los estudiantes manipulan los ordenadores mientras el } \\
\text { profesor, oralmente, indica hacia dónde deben dirigirse para llegar a algún } \\
\text { sitio, lo que deben hacer para elaborar un producto o descargar algún texto } \\
\text { para leerlo. }\end{array}$ \\
\hline $\begin{array}{l}\text { SADE5 } \\
\text { Realizar } \\
\text { ejercicios }\end{array}$ & $\begin{array}{l}\text { Fragmentos en que los estudiantes completan o contestan preguntas. Para } \\
\text { ello, no necesariamente deben usar información extraída de la web, sino más } \\
\text { bien lo que saben (conocimientos previos) o bien documentos que han sido } \\
\text { entregados o indicados por parte del profesor. Por tanto, aquí la TIC cumple } \\
\text { un rol de instrumento evaluativo, pues en ella está contenido un cuestionario } \\
\text { que los estudiantes responden y que servirá al profesor para evaluar en alguna } \\
\text { medida los logros. La TIC es un contenedor de respuestas emitidas por los } \\
\text { estudiantes sobre algunos temas de los trabajados durante la SD. }\end{array}$ \\
\hline
\end{tabular}




\begin{tabular}{|l|l|}
\hline $\begin{array}{l}\text { SADE6 } \\
\text { Elaborar } \\
\text { productos de } \\
\text { aprendizaje }\end{array}$ & $\begin{array}{l}\text { SAD en que los estudiantes deben elaborar un producto o material usando las } \\
\text { TIC. Corresponde a una tarea que les asigna el profesor cuya ejecución } \\
\text { implica hacer algo específico usando alguna TIC, por ejemplo, una tabla en } \\
\text { Word o un blog. Por tanto, el foco aquí no está en la búsqueda de información, } \\
\text { sino en que dicha búsqueda sirva para hacer algo con ella, en específico, el } \\
\text { objetivo de la actividad se centra en dicho producto, aunque para hacerlo han } \\
\text { de buscar y seleccionar información principalmente de Internet. }\end{array}$ \\
\hline $\begin{array}{l}\text { SADE7 } \\
\text { Apoyar una } \\
\text { disertación }\end{array}$ & $\begin{array}{l}\text { Fragmentos de la SD que corresponden al momento en que los estudiantes } \\
\text { exponen su trabajo a sus compañeros apoyados de algún recurso TIC con } \\
\text { preparación tipo "disertación". En estos segmentos el profesor asume una } \\
\text { actitud pasiva y entrega a los estudiantes el protagonismo, pues ellos exponen, } \\
\text { apoyados preferentemente de un PowerPoint o de sus productos elaborados } \\
\text { en un blog, procesador de texto o Paint. El propósito en este caso es dar } \\
\text { cuenta por parte de los estudiantes de los productos solicitados y del trabajo } \\
\text { llevado a cabo durante una sesión o bien durante toda la SD. }\end{array}$ \\
\hline
\end{tabular}

Fuente: Arancibia, Soto y Casanova (2017, p.107).

En síntesis, los SAD que se han encontrado en las trece SD dan cuenta de una homogeneidad de usos de parte de profesores y estudiantes. Los cuatro SADP se concentran básicamente en modos de pasar contenidos o modelar productos, en otras palabras, se usa un recurso previamente creado ya sea por el profesor (PowerPoint), por sus estudiantes (blog) o agentes externos (video). En tanto, los 7 SADE en que los estudiantes usan las TIC se caracterizan por ser tipos de uso básicamente referidos a navegación en la Web o en recursos para apoyar exposiciones, con una baja presencia, por un lado, de usos creativos orientados a la publicación o construcción de ambientes digitales (Sigalès, Mominó, Meneses y Badia, 2009) y, por otro lado, de usos comunicativos encaminados a la publicación y discusión de los contenidos o uso de redes sociales (Cobo y Moravec, 2011).

Observamos que en todas las SD el profesor tiene un bajo uso de las TIC, particularmente destaca P11 que no usa en ningún momento las TIC, le sigue P3 con solo un 2,3\% del tiempo en que las TIC son utilizadas directamente por él, luego P8 con solo un 2,4\% de uso. En el lado opuesto, se encuentra P2 que las usa en un 40,5\%, seguido de P7 con un $31,6 \%$ de uso de TIC. Mientras que presentan porcentajes de uso muy similares, por un lado, P1 y P4 (11,1\% y 12,5\% respectivamente), y por otro P5 y P10 (28,6\% y 29,6\% respectivamente). Todos los profesores del estudio facilitan el acceso al contenido por parte de los alumnos, sin embargo, existen diversos perfiles de actuaciones didácticas, de más a menos control por parte del profesor y en cuanto al tipo de tarea que se les asigna a los estudiantes, ello se resume en el Cuadro 7: 
Estudios Pedagógicos XLV, Nº 1: 103-121, 2019

RELACIONES ENTRE CONCEPCIONES Y PRÁCTICAS PEDAGÓGICAS: ANÁLISIS DE 13 SECUENCIAS DIDÁCTICAS DE PROFESORES DE HISTORIA USANDO TECNOLOGÍAS EN EL AULA ESCOLAR

Cuadro 7. Perfiles de uso didáctico de TIC de los profesores

\begin{tabular}{|l|l|l|}
\hline & SADP & SADE \\
\hline (P1, P6, P11) & & Elaborar productos + Buscar información \\
\hline (P2) & Exposición oral & Realizar ejercicios + Acceder a información seleccionada \\
\hline (P3) & & Buscar información + Acceder a información seleccionada \\
\hline (P4) & & Buscar información + Apoyar una disertación \\
\hline (P5) & Exposición oral & Buscar información + Apoyar una disertación \\
\hline (P7) & Apoyan conversación & Realizan ejercicios + Elaborar productos \\
\hline (P8) & & Elaborar productos + Apoyar una disertación \\
\hline (P9) & & Elaborar productos + Realizar ejercicios + Buscar información \\
\hline (P10) & Exposición oral & Elaborar productos + Buscar información \\
\hline (P12) & Exposición oral & Realizar ejercicios + Acceder a información seleccionada \\
\hline (P13) & Muestra ejemplos & Elaborar productos + Buscar información \\
\hline
\end{tabular}

Fuente: Propia

En primer lugar, se puede indicar que P1, P6 y P11 usan las TIC preferentemente con similar intencionalidad. En los perfiles del cuadro 7, se observa que mayoritariamente los estudiantes elaboran productos con las TIC, en algunos casos junto con la búsqueda de información (P1, P6, P9, P10, P11 y P13) o para apoyar una exposición (P8); en este último caso, los estudiantes no usan las TIC para buscar información en clases, ellos la traen y trabajan en el producto que luego expondrán (diaporama). En igual sentido, P7 combina la realización de ejercicios en que contestan preguntas o cuestionarios específicos, en este caso el análisis de una planilla Excel con gráficos de población, y luego la elaboración de un producto (mapa conceptual). Es llamativa también la SD de P13, en la cual el profesor usa las TIC para mostrar ejemplos, es decir exhibir modelos. Se diferencian en este análisis los perfiles de P4 y P5, pues sus prácticas educativas con TIC se orientan a buscar información y luego apoyar la exposición de los estudiantes. En las clases de estos profesores no se observa que los estudiantes preparen el producto de su presentación, es más, este queda como tarea asignada antes de las sesiones en que exponen. En los perfiles de P2, P5, P10 y P12, se evidencia uso de las TIC para apoyar la exposición del profesor. En tanto P7 es el único que muestra en su perfil un uso de parte del profesor para apoyar las conversaciones con los estudiantes.

Clasificados los profesores en concepción preferente y descritas sus prácticas con uso de TIC, en el cuadro 8, para responder a nuestra pregunta, se muestra para cada profesor las relaciones entre concepciones preferentes en cada Dimensión y los SAD más utilizados en las SD. 
Estudios Pedagógicos XLV, N $^{\circ}$ 1: 103-121, 2019 RELACIONES ENTRE CONCEPCIONES Y PRÁCTICAS PEDAGÓGICAS: ANÁLISIS DE 13 SECUENCIAS DIDÁCTICAS DE PROFESORES DE HISTORIA USANDO TECNOLOGÍAS EN EL AULA ESCOLAR

Cuadro 8. Relación y asociación entre concepción y SAD más usados por cada profesor

\begin{tabular}{|c|c|c|c|c|}
\hline & \multicolumn{3}{|c|}{ Concepción preferente por Dimensión } & \multirow[b]{2}{*}{ SAD más utilizados por cada profesor } \\
\hline & Curriculum & $\begin{array}{l}\text { Acción } \\
\text { didáctica }\end{array}$ & $\begin{array}{l}\text { Tecno/ } \\
\text { didáctica }\end{array}$ & \\
\hline P1 & Crítico & $\begin{array}{l}\text { Constructivista/ } \\
\text { social }\end{array}$ & $\begin{array}{l}\text { Constructivista/ } \\
\text { social }\end{array}$ & $\begin{array}{l}\text { SADE6 Elaboran productos de aprendizaje }(37,5 \%) \\
\text { SADE3 Buscan información y documentos }(27,4 \%) \\
\text { SADE7 Apoyan una disertación }(12,1 \%) \\
\text { SADP2 Apoya conversaciones con alumnos }(9,4 \%)\end{array}$ \\
\hline P3 & Crítico & $\begin{array}{l}\text { Constructivista/ } \\
\text { social }\end{array}$ & $\begin{array}{l}\text { Constructivista/ } \\
\text { individual }\end{array}$ & $\begin{array}{l}\text { SADE3 Buscan información y documentos }(73,5 \%) \\
\text { SADE1 Acceden a información seleccionada }(14,9 \%) \\
\text { SADE7 Apoyan una disertación }(12,1 \%)\end{array}$ \\
\hline P7 & Crítico & $\begin{array}{l}\text { Constructivista/ } \\
\text { social }\end{array}$ & Múltiple & $\begin{array}{l}\text { SADE5 Realizan ejercicios }(35 \%) \\
\text { SADE6 Elaboran productos de aprendizaje }(26,2 \%) \\
\text { SADP2 Apoya conversaciones con los alumnos } \\
(21,2 \%)\end{array}$ \\
\hline P11 & Crítico & $\begin{array}{l}\text { Constructivista/ } \\
\text { social }\end{array}$ & $\begin{array}{l}\text { Constructivista/ } \\
\text { individual }\end{array}$ & $\begin{array}{l}\text { SADE3 Buscan información y documentos }(73,5 \%) \\
\text { SADE4 Seguir instrucciones }(2,6 \%)\end{array}$ \\
\hline $\mathbf{P 2}$ & Técnico & $\begin{array}{l}\text { Transmisiva/ } \\
\text { reproductiva }\end{array}$ & $\begin{array}{l}\text { Transmisiva/ } \\
\text { reproductiva }\end{array}$ & $\begin{array}{l}\text { SADP1 Apoyo a su exposición Oral }(36,9 \%) \\
\text { SADE5 Realizan ejercicios }(29,2 \%) \\
\text { SADE1 Acceden a información seleccionada }(14,9 \%)\end{array}$ \\
\hline P4 & Técnico & $\begin{array}{l}\text { Constructivista/ } \\
\text { individual }\end{array}$ & $\begin{array}{l}\text { Transmisiva/ } \\
\text { reproductiva }\end{array}$ & $\begin{array}{l}\text { SADE3 Buscan información y documentos }(65,1 \%) \\
\text { SADP1 Apoyo a su exposición Oral }(12,5 \%) \\
\text { SADE7 Apoyan una disertación }(18,5 \%)\end{array}$ \\
\hline P5 & Técnico & $\begin{array}{l}\text { Transmisiva/ } \\
\text { reproductiva }\end{array}$ & $\begin{array}{l}\text { Transmisiva/ } \\
\text { reproductiva }\end{array}$ & $\begin{array}{l}\text { SADE3 Buscan información y documentos }(40,5 \%) \\
\text { SADP1 Apoyo a su exposición Oral }(28,6 \%) \\
\text { SADE7 Apoyan una disertación. }(22,5 \%)\end{array}$ \\
\hline P10 & Técnico & $\begin{array}{l}\text { Transmisiva/ } \\
\text { reproductiva }\end{array}$ & $\begin{array}{l}\text { Constructivista/ } \\
\text { social }\end{array}$ & $\begin{array}{l}\text { SADE6 Elaboran productos de aprendizaje }(30,6 \%) \\
\text { SADP1 Apoyo a su exposición oral }(21,9 \%) \\
\text { SADE5 Realizan ejercicios }(20,8 \%)\end{array}$ \\
\hline P13 & Técnico & $\begin{array}{l}\text { Transmisiva/ } \\
\text { reproductiva }\end{array}$ & $\begin{array}{l}\text { Transmisiva/ } \\
\text { reproductiva }\end{array}$ & $\begin{array}{l}\text { SADE6 Elaborar productos de aprendizaje }(36,3 \%) \\
\text { SADE3 Buscan información y documentos }(32 \%)\end{array}$ \\
\hline P6 & Práctico & $\begin{array}{l}\text { Constructivista/ } \\
\text { individual }\end{array}$ & $\begin{array}{l}\text { Constructivista/ } \\
\text { individual }\end{array}$ & $\begin{array}{l}\text { SADE3 Buscan información y documentos }(53 \%) \\
\text { SADE6 Elaboran productos de aprendizaje }(34,4)\end{array}$ \\
\hline P8 & Práctico & $\begin{array}{l}\text { Constructivista/ } \\
\text { individual }\end{array}$ & $\begin{array}{l}\text { Constructivista/ } \\
\text { individual }\end{array}$ & $\begin{array}{l}\text { SADE6 Elaboran productos de aprendizaje }(63,5 \%) \\
\text { SADE7 Apoyan una disertación. }(32,2 \%)\end{array}$ \\
\hline P9 & Práctico & $\begin{array}{l}\text { Constructivista/ } \\
\text { individual }\end{array}$ & $\begin{array}{l}\text { Constructivista/ } \\
\text { individual }\end{array}$ & $\begin{array}{l}\text { SADE6 Elaboran productos de aprendizaje }(30,6 \%) \\
\text { SADE5 Realizan ejercicios }(20,8 \%) \\
\text { SADP1 Apoyo a su exposición oral }(15,1 \%)\end{array}$ \\
\hline P12 & Crítico & $\begin{array}{l}\text { Constructivista/ } \\
\text { individual }\end{array}$ & $\begin{array}{l}\text { Constructivista/ } \\
\text { individual }\end{array}$ & $\begin{array}{l}\text { SADE3 Buscan información y documentos }(43,5 \%) \\
\text { SADE5 Realizan ejercicios }(33,9 \%)\end{array}$ \\
\hline
\end{tabular}


Estudios Pedagógicos XLV, № 1: 103-121, 2019

RELACIONES ENTRE CONCEPCIONES Y PRÁCTICAS PEDAGÓGICAS: ANÁLISIS DE 13 SECUENCIAS DIDÁCTICAS DE PROFESORES DE HISTORIA USANDO TECNOLOGÍAS EN EL AULA ESCOLAR

En el caso de las relaciones entre P1, P3, P7 y P11 (grupo preferentemente de concepción social constructivista), existe un correlato entre sus concepciones y los SAD mayormente presentes en sus SD. En primer lugar, las concepciones de estos profesores identifican un curriculum crítico marcado por una práctica docente que busca motivar a los estudiantes y un uso de TIC transformador; mientras que sus SAD están orientados a generar diálogos entre y con los estudiantes, a que sean ellos los que busquen información para elaborar productos, los cuales luego son presentados y discutidos. Estos profesores (salvo P7) no usan las TIC para exponer contenidos, por tanto, estas son más bien un medio para establecer una relación con los estudiantes y de estos con el contenido.

Por su parte, la relación expuesta en el cuadro 8, entre P2, P4, P5, P10 y P13 (grupo preferentemente de concepción transmisiva reproductiva) es sin duda la más consistente, presentando un alto nivel de cercanía entre sus concepciones con sus SAD. Por un lado, tienen una concepción técnica sobre el currículo y una concepción en relación a la acción didáctica y al uso educativo de las TIC de tipo transmisiva/reproductiva (salvo P10 que en M3 es constructivista), concepciones que se ven refrendadas en su práctica por el hecho de que sus SAD más frecuentes y con mayor tiempo corresponden a apoyar la exposición de contenidos del profesor a los estudiantes, en cuyo caso el uso de TIC está en relación con buscar información y realizar ejercicios.

En tal sentido, un profesor de concepción técnica del currículo o externa/transmisiva de la acción didáctica muestra una tendencia a exponer mucho contenido con uso de TIC, por tanto, hay un alto porcentaje de tiempo en que él usa las TIC (SADP). También son profesores, a excepción de P5, que no demuestran mucho interés en que los estudiantes den cuenta de sus trabajos, no hay SAD de exposiciones, es más cuando lo hace P5 su actitud es pasiva, sin mediar comentarios o reflexiones sobre lo que los estudiantes exponen.

En cuanto a las relaciones establecidas entre P6, P8, P9 y P12 (grupo preferentemente de concepción constructivista individual), es posible encontrar también en los SAD de sus SD, en particular en cuanto a que los estudiantes están permanentemente elaborando productos de aprendizaje. Los usos de las TIC, están principalmente entregados a los estudiantes casi con total autonomía (salvo P9 que presenta un porcentaje importante de exposición oral). En este sentido, el currículo entendido como práctico supone su adecuación a los intereses de sus estudiantes, a su vez, que estos aprendan haciendo. Por su parte, en cuanto a la acción didáctica, concebida como constructiva individual, podemos ver que igualmente estos profesores cuando proponen usar las TIC lo hacen con la intención de que se elaboren productos de aprendizaje que luego son expuestos a sus compañeros (salvo P6 que no exhibe SADE2).

Dadas las relaciones establecidas, cabe consignar que todas las SD son diferentes, no existen dos iguales o incluso parecidas, más bien lo que es posible encontrar es la presencia similar de algunos SAD en las respectivas SD. Incluso, esta diferencia se observa dentro de los agrupamientos que hemos realizado de los profesores por similar concepción preferente.

\section{CONCLUSIONES}

Realizada la relación entre concepciones preferentes y uso de TIC de los 13 profesores, podemos aseverar que quienes presentan concepciones similares tienden a organizar desde 
un punto de vista estructural su secuencia didáctica de modo parecido en los segmentos en que el profesor las usa (SADP), sin embargo, este vínculo no es tan nítido en aquellos en que las usan los estudiantes (SADE). Se ha demostrado que las relaciones entre concepciones y las prácticas con uso de tecnología se dan a ciertos niveles dentro de las respectivas secuencias didácticas. Es decir, existe incidencia de las concepciones acerca de aprender y enseñar historia sobre los usos educativos de las tecnologías en profesores de concepciones transmisiva y constructivistas.

Otra conclusión establece que el tipo de concepciones sobre enseñar y aprender que tenga el profesor influye en cómo se reparte el tiempo de uso de las tecnologías entre el propio profesor y sus alumnos. Los profesores con una concepción transmisiva, tienden a usar más tiempo el ordenador, mientras que los profesores con una concepción de orden constructivista exhiben las SD con mayor uso de tecnología de parte de los estudiantes.

La principal conclusión a la que llega esta investigación es que existe una interrelación posible de establecer entre concepciones sobre enseñar y aprender, y prácticas docentes con la tecnología. Esta conexión entre concepciones y uso de la tecnología se evidencia en los patrones de usos establecidos por grupos de profesores según concepción preferente. Ello nos hace suponer que los usos educativos de las tecnologías están integrados dentro del sistema didáctico de un profesor como un elemento en alguna medida supeditado a sus concepciones sobre enseñar y aprender. Estas concepciones operan como un conocimiento regulador que prevalece y es utilizado como uno de los criterios que considera el profesor al momento de seleccionar qué tipos de tecnologías son asumibles, por tanto, coherentes con un tipo de concepción, y también cómo serán usadas estas tecnologías para poner en juego una determinada intencionalidad educativa en las aulas.

Respecto de esto, la toma de decisiones didácticas que efectúa un profesor está influenciada por sus concepciones, de allí que emerja como fundamental el conocimiento explícito de estas concepciones si lo que se pretende es cambiar una práctica pedagógica. El alcance de este planteamiento se extiende más allá del uso de computadores en contextos escolares y se expresa como aspecto fundamental a trabajar en la formación de profesores.

Una de las cuestiones más controversiales en torno al cambio educativo tiene que ver con la reproducción de las prácticas escolares, es decir, se viene haciendo siempre lo mismo desde hace mucho tiempo. Con ello, la incorporación de TIC como artefactos que sirvan a la instalación de procesos innovadores que promuevan la renovación de las prácticas es algo que no ha tenido el éxito pronosticado. Nuestra investigación entrega antecedentes relevantes en función de que si bien los profesores otorgan en el uso de las TIC mayor protagonismo a los estudiantes, no logran llevar a cabo prácticas constructivistas (innovadoras) con estas, lo cual se debe, entre otros factores, a que sus estructuras didácticas siguen ancladas en paradigmas tradicionales. Con ello, podemos destacar que las TIC en sí mismas no son artefactos que cambian las prácticas, en este sentido, se debe dar en el sistema escolar un paso más allá del simple acto de instalar ordenadores o de promover por decreto prácticas innovadoras.

Un aporte de estos resultados es instar a que la instalación de TIC en las escuelas y la facilitación de usos innovadores se deban relacionar con las concepciones docentes y con los contextos de ejercicio profesional, de modo que efectivamente se pueda cumplir con el anhelo de transformar la enseñanza hacia modelos constructivistas con uso de tecnología, en las cuales las tecnologías pasan a ser un medio para modificar también las concepciones pedagógicas tradicionales. 
Estudios Pedagógicos XLV, $\mathrm{N}^{\circ}$ 1: 103-121, 2019

RELACIONES ENTRE CONCEPCIONES Y PRÁCTICAS PEDAGÓGICAS: ANÁLISIS DE 13 SECUENCIAS DIDÁCTICAS DE PROFESORES DE HISTORIA USANDO TECNOLOGÍAS EN EL AULA ESCOLAR

\section{REFERENCIAS BIBLIOGRÁFICAS}

Arancibia, M. y Badia, A. (2013). Caracterización y valoración de los usos educativos de las TIC en 10 secuencias didácticas de historia en enseñanza secundaria. Estudios Pedagógicos, 39(Especial), 7-24.

Arancibia, M. y Badia, A. (2015). Concepciones de profesores de secundaria sobre enseñar y aprender Historia con TIC. Revista Electrónica de Investigación Educativa, 17(2), 62-76.

Arancibia, M., Soto, C. y Casanova, R. (2017). Análisis de los segmentos de actuación docente en diez secuencias didácticas de profesores de historia usando tecnologías. Diálogo Andino, (52), $101-115$.

Arancibia, M., Badia, A., Soto, C. y Sigerson, A. (2018). The impact of secondary history teachers' teaching conceptions on the classroom use of computers. Technology, Pedagogy and Education, 27(1), 101-114. doi:10.1080/1475939X.2017.1412342

Bloch, M. (1975). Introducción a la historia. México DF: Fondo de Cultura Económica.

Camillioni, A. (1994). Epistemología de la didáctica de las ciencias sociales. En Aisenberg, B. y Alderoqui, S. (Eds.), Didáctica de las ciencias sociales. Aportes y reflexiones (pp. 11-25). Buenos Aires: Paidos.

Cobo, C. y Moravec, J. (2011). Aprendizaje invisible. Barcelona: Editorial UAB.

Coll, C., Rochera, M. \& Colomina, R. (2010). Situated uses of ICT and mediation of Joint activity in a primary education instructional sequence. Electronic Journal of Research in Educational Psychology, 8(2), 517-540.

Collingwood, R. (1977). Idea de la historia. México DF: Fondo de Cultura Económica.

Ertmer, P. \& Ottenbreit-Leftwich, A. (2010). Teacher Technology Change: How Knowledge, Confidence, Beliefs, and Culture Intersect. Journal of Research on Technology in Education, 42(3), 255-284.

Estepa, J. (2000). La investigación sobre el conocimiento profesional de los Profesores para enseñar ciencias sociales. Ponencia presentada al XI simposium internacional de didáctica de las ciencias sociales. Huelva - España. Recuperado de: http://goo.gl/56Jt4 [20/11/2011].

Evans, R. (1994). Educational ideologies and the teaching of history. In G. Leinhardt, E. Beck, \& C. Stainton (Eds.), Learning and teaching in history (pp. 171-208). Hillsdale, NJ: Lawrence Erlbaum.

Flick, U. (2004). Introducción a la investigación cualitativa. Madrid: Morata.

Friera, F. (1995). Didáctica de las ciencias sociales. Geografía e Historia. Madrid: Ediciones de la Torre.

Gutiérrez, A. y Tyner, K. (2012). Educación para los medios, alfabetización mediática y competencia digital. Comunicar, 19(38), 31-39.

Haydn, T. (2011). Secondary history: Current themes. In I. Davies (Ed.), Debates in history teaching (pp. 30-45). Abingdon: Routledge.

Inan, F., Lowther, D., Ross, S. \& Strahl, D. (2010) Pattern of classroom activities during students' use of computers: Relations between instructional strategies and computer applications. Teaching and Teacher Education, 26, 540-546. doi:10.1016/j.tate.2009.06.017.

Maggioni, L., VanSledright, B. \& Alexander, P. (2009). Walking on the borders: A measure of epistemic cognition in history. The Journal of Experimental Education, 77(3), 187-213. doi:10.3200/JEXE.77.3.187-214

Marton, F. y Booth, Sh. (1997). Learning and awareness. Mahwah: Routledge.

McCrum, E. (2013). History teachers' thinking about the nature of their subject. Teaching and Teacher Education, 35, 73-80. http://dx.doi.org/10.1016/j.tate.2013.05.004

Mueller, J., Wooda, E., Willoughby, T., Ross, C. \& Specht, J. (2008). Identifying discriminating variables between teachers who fully integrate computers and teachers with limited integration. Computers \& Education, 51, 1523-1537. 
Petko, D. (2012). Teachers' pedagogical beliefs and their use of digital media in classrooms: Sharpening the focus of the 'will, skill, tool' model and integrating teachers' constructivist orientations. Computers \& Education, 58, 1351-1359, doi:10.1016/j.compedu.2011.12.013.

Sigalès, C., Mominó, J., Meneses, J. y Badia, A. (2009). La integración de internet en la educación escolar española. Barcelona: Ariel.

Stake, R. (1999). Investigación con Estudios de Casos.Madrid: Ediciones Morata.

Starkey, L. (2010). Teachers' pedagogical reasoning and action in the digital age. Teachers and Teaching: Theory and Practice, 16(2), 233-244.

Starkey, L. (2011). Evaluating learning in the 21st century: a digital age learning matrix, Technology. Pedagogy and Education, 20(1), 19-39.

Tigchelaar, A., Hermans, R., Van Braak, J. \& Valcke, N. (2012). Patterns of development in secondcareer teachers' conceptions of learning and teaching. Teaching and Teacher Education, 28, 1163-1174. doi:10.1016/j.tate.2012.07.006.

Tondeur, J., Hermans, R., Van Braak, J. \& Valcke, M. (2008). Exploring the link between teachers' educational belief profiles and different types of computer use in the classroom. Computers in Human Behaviour, 24, 2541-2553. doi:10.1016/j.chb.2008.02.020.

Voet, M. \& de Wever, B. (2016). History teachers' conceptions of inquiry-based learning, beliefs about the nature of history, and their relation to the classroom context. Teaching and Teacher Education, 55, 57-67. http://dx.doi.org/10.1016/j.tate.2015.12.008

Yilmaz, K. (2010). Social studies teachers' conceptions of history: Calling on historiography. Journal of Educational Research, 101(3), 37-41. doi:10.3200/JOER.101.3.158-176. 
